\title{
Multifunctional polarization tomography of birefringence networks of biological crystals
}

\author{
O.V. Dubolazov ${ }^{1}$, O.I. Olar ${ }^{2}$, D.M. Burkovets ${ }^{1}$ \\ ${ }^{1}$ Chernivtsi National University, Optics and Publishing Department \\ 2, Kotsyubinsky str., 58012 Chernivtsi, Ukraine \\ ${ }^{2}$ Bukovinian State Medical University, 58000 Chernivtsi, Ukraine \\ E-mail: a.dubolazov@chnu.edu.ua
}

\begin{abstract}
The results of optical modeling of biological crystals polycrystalline multilayer networks have been presented. Determined in this work are the algorithms of reconstruction of parameter distributions that describe the linear and circular birefringence. To separate manifestations of these mechanisms, we propose the method of space-frequency filtering. Criteria for differentiation of benign and malignant tissues of the women reproductive sphere have been found.
\end{abstract}

Keywords: polarization tomography, biological crystals, diagnostics.

Manuscript received 06.11.14; revised version received 25.02.15; accepted for publication 27.05.15; published online 08.06.15.

\section{Introduction}

In numerous areas of optical diagnostics of biological tissue structures, the techniques based on polarized laser radiation are widely used [1-3]. Particularly, laser polarimetry of microscopic images of polycrystalline protein networks was formed as a separate approach in the study of optically anisotropic component of different biological tissues biopsy [4-28].

Our research is aimed at designing the experimental method of Fourier polarimetry and spatialfrequency selection of parameter distributions of linear and circular birefringence of the biopsy of the uterine wall tissue in order to differentiate benign (fibromyoma) and malignant (adenocarcinoma) conditions.

\section{Theory of the method}

The following model concepts have been assumed [14] by us as the basis for an analysis of the processes of amplitude-phase modulation of laser radiation by the polycrystalline network of biological crystals with linear and circular birefringence:

- the uterine wall tissue consists of two optically anisotropic layers of the muscular (myometrium) and connective (endometrium) layers;
- phase anisotropy of these layers formed by optically active myosin and collagen proteins that form fibrillar network with different conformational structure;

- $\quad$ the optically anisotropic myometrium network is formed by spatially ordered myosin fibrils. This spatial organization generates large-scale (the range of the transverse dimensions $l \sim 50 \ldots 300 \mu \mathrm{m}$ ) optically uniaxial birefringent linear structure. Polarization properties of biological crystals are characterized by the Jones matrix of the following form

$\{Q\}=\left\|\begin{array}{ll}\sin ^{2} \rho+\cos ^{2} \rho \exp (-i \delta) ; & \sin \rho \cos \rho(1-\exp (-i \delta)) \\ \sin \rho \cos \rho(1-\exp (-i \delta)) ; & \cos ^{2} \rho+\sin ^{2} \rho \exp (-i \delta)\end{array}\right\|$,

where $\rho$ is the direction of the axes, it determines the direction of stacking fibrils; $\delta=\frac{2 \pi l}{\lambda} \Delta n$ is the value of the phase shift between orthogonal components of the amplitude; $\lambda$ - wavelength; $\Delta n$ - linear birefringence;

- the optically anisotropic component of the endometrium is formed by small-scale $(l \sim$ $5 . .25 \mu \mathrm{m})$ randomly oriented collagen fibrils with predominant circular birefringence 
$\{A\}=\left\|\begin{array}{cc}\cos \theta & \sin \theta \\ -\sin \theta & \cos \theta\end{array}\right\|$,

where $\theta=\frac{\pi l}{\lambda} \Delta n_{ \pm}$is the angle of the polarization plane; $\Delta n_{ \pm}$- indicator of the circular birefringence.

- the process of converting laser radiation by optically anisotropic fibrils is characterized by the following matrix equation

$E=\{Q\}\{A\} E_{0}$.

The coordinate distribution of parameters describing linear $\delta$ and circular $\theta$ anisotropy of fibrillar network layers of the myometrium and endometrium can be found from the corresponding Jones matrix (relations (4) and (5)).

$$
\begin{aligned}
& \delta\left(q_{i k}\right)=\arccos \left\{\frac{i\left(E_{y} E_{x}^{*}-E_{x} E_{y}^{*}\right)_{\otimes}}{\left(E_{y} E_{x}^{*}+E_{x} E_{y}^{*}\right)_{\otimes}}-\right. \\
& \left.-0.5 i\left[\frac{\left(E_{y} E_{x}^{*}-E_{x} E_{y}^{*}\right)_{0}}{\left(E_{y} E_{x}^{*}+E_{x} E_{y}^{*}\right)_{0}}+\frac{\left(E_{y} E_{x}^{*}-E_{x} E_{y}^{*}\right)_{90}}{\left(E_{y} E_{x}^{*}+E_{x} E_{y}^{*}\right)_{90}}\right]\right\}, \\
& \theta\left(a_{i k}\right)=\arccos \left\{\frac{\left(E_{x} E_{x}^{*}-E_{y} E_{y}^{*}\right)_{0}}{\left(E_{x} E_{x}^{*}+E_{y} E_{y}^{*}\right)_{0}}-\frac{\left(E_{x} E_{x}^{*}-E_{y} E_{y}^{*}\right)_{90}}{\left(E_{x} E_{x}^{*}+E_{y} E_{y}^{*}\right)_{90}}\right\} .
\end{aligned}
$$

Here, we use the following notations:

- $\quad *$ - complex conjugate;

- $\otimes-$ sensing the right circularly polarized wave $E_{0}=\left(\begin{array}{l}1 \\ i\end{array}\right)$

- 0 - sounding the linearly polarized wave $E_{0}=\left(\begin{array}{l}1 \\ 0\end{array}\right)$ with azimuth $0^{\circ}$;

- 90 - sounding the linearly polarized wave $E_{0}=\left(\begin{array}{l}0 \\ 1\end{array}\right)$ with azimuth $90^{\circ}$.

From the medical point of view, "optic separation" of various anisotropy mechanisms in layers of the uterine wall is the topical task. The fact is that at the initial, early stages, the pathological changes are accompanied by a concentration increase in the amount of optically active proteins in polycrystalline network of the myometrium and endometrium. Fibrillar structural changes - the formation of the growth direction of tumour shoots and so on - correspond to a more developed pathological condition.

With the purpose to select these conditions, we have applied the method of spatial-frequency filtration of the polarization-inhomogeneous field $E_{x}(\rho, \delta, \theta)$ and $E_{y}(\rho, \delta, \theta)$ in Fourier plane. The main idea of this approach is that spatial-frequency structure of Fourier form of laser image inherent to the tissue of the uterine wall is different for its large-scale (myosin) and smallscale (collagen) protein structures. Proceeding from this, using spatial-frequency filtration, it is mainly possible to isolate either low-frequency (with linear birefringence) or high-frequency (with circular birefringence) components, which by means of Fourier conversion can be transformed into respectively "separated" laser images.

In our case, we consider the situation when the distance between projection lens, the layer of the object under study, and the plane of registration constitute the focal distance $f$.

$$
\begin{aligned}
& U_{x}(\nu, \mu)=\frac{1}{i \lambda f} \int_{-\infty}^{\infty} \int_{x} E_{x}(x, y) \exp [-i 2 \pi(x v+y \mu)] d x d y, \\
& U_{y}(\nu, \mu)=\frac{1}{i \lambda f} \int_{-\infty}^{\infty} \int_{y} E_{y}(x, y) \exp [-i 2 \pi(x v+y \mu)] d x d y .
\end{aligned}
$$

Here, $U_{x}, U_{y}$ are Fourier images of distributions $E_{x}(\rho, \delta, \theta)$ and $E_{y}(\rho, \delta, \theta)$ in the focal plane with spatial frequencies $\nu=\frac{x}{\lambda f}$ and $\mu=\frac{y}{\lambda f}$.

If we place vignetting transparent $R(\Delta v, \Delta \mu)$ or opaque $R^{-1}(\Delta v, \Delta \mu)$ diaphragm in the central part of Fourier plane, we can identify corresponding areas of the spatial-frequency structure of the distribution amplitudes, which are formed by predominant influences of linear $\hat{U}(\rho, \delta, \nu, \mu)$ and circular $\dot{U}(\theta, \nu, \mu)$ birefringence

$\left\{\begin{array}{l}\hat{U}(\rho, \delta, \nu, \mu)=R(\Delta v, \Delta \mu) U(\nu, \mu) \\ \dot{U}(\theta, \nu, \mu)=R^{-1}(\Delta \nu, \Delta \mu) U(\nu, \mu) .\end{array}\right.$

To renew the proper distributions of complex amplitudes $\left\{\begin{array}{l}\hat{E}_{x}(\rho, \delta, x, y) \\ \dot{E}_{x}(\theta, x, y)\end{array}\right.$ and $\left\{\begin{array}{l}\hat{E}_{y}(\rho, \delta, x, y) \\ \dot{E}_{y}(\theta, x, y)\end{array}\right.$ in the image plane of this layer is possible by reverse Fourier transformation

$$
\left\{\begin{array}{l}
\hat{E}_{x}(\rho, \delta, x, y)= \\
=\frac{1}{i \lambda f} \iint_{-\infty}^{\infty} R(\Delta v, \Delta \mu) \hat{U}_{x}(\nu, \mu) \exp [i 2 \pi(x \Delta \nu+y \Delta \mu)] d v d \mu, \\
\dot{E}_{x}(\theta, x, y)= \\
=\frac{1}{i \lambda f} \int_{-\infty}^{\infty} \int_{0}^{-1}(\Delta v, \Delta \mu) \dot{U}_{x}(\nu, \mu) \exp [i 2 \pi(x \Delta v+y \Delta \mu)] d v d \mu,
\end{array}\right.
$$

$$
\left\{\begin{array}{l}
\hat{E}_{y}(\rho, \delta, x, y)= \\
=\frac{1}{i \lambda f} \iint_{-\infty}^{\infty} R(\Delta v, \Delta \mu) \hat{U}_{y}(\nu, \mu) \exp [i 2 \pi(x \Delta v+y \Delta \mu)] d v d \mu, \\
\dot{E}_{y}(\theta, x, y)= \\
=\frac{1}{i \lambda f} \int_{-\infty}^{\infty} \int^{-1}(\Delta v, \Delta \mu) \dot{U}_{y}(\nu, \mu) \exp [i 2 \pi(x \Delta v+y \Delta \mu)] d v d \mu .
\end{array}\right.
$$

\section{(C) 2015, V. Lashkaryov Institute of Semiconductor Physics, National Academy of Sciences of Ukraine}


Within each pixel of a digital camera kits measured intensities $R \rightarrow I_{\otimes}^{\otimes} ; \quad I_{\oplus}^{\otimes} ; \quad I_{\otimes}^{0} ; \quad I_{\oplus}^{0} ; \quad I_{\otimes}^{90} ; \quad I_{\oplus}^{90} \quad$ and $R^{-1} \rightarrow I_{0}^{0} ; \quad I_{90}^{0} ; \quad I_{0}^{90} ; \quad I_{90}^{90}$. The magnitude of phase shift $\delta(R)$ and a rotation of plane polarization $\theta\left(R^{-1}\right)$ are calculated according to the following equations.

$$
\begin{aligned}
& \delta(R)=\arccos \left\{\frac{\left(I_{\circledast}^{\otimes}-I_{\oplus}^{\otimes}\right)}{\left(I_{\circledast}^{\otimes}+I_{\oplus}^{\otimes}\right)}-\right. \\
& \left.-0.5\left[\frac{\left(I_{\circledast}^{0}-I_{\oplus}^{0}\right)}{\left(I_{\circledast}^{0}+I_{\oplus}^{0}\right)}+\frac{\left(I_{\circledast}^{90}-I_{\oplus}^{90}\right)}{\left(I_{\circledast}^{90}+I_{\oplus}^{90}\right)}\right]\right\},
\end{aligned}
$$

$$
\theta\left(R^{-1}\right)=0.5 \arccos \left\{\frac{\left(I_{0}^{0}-I_{90}^{0}\right)}{\left(I_{0}^{0}+I_{90}^{0}\right)}-\frac{\left(I_{0}^{90}-I_{90}^{90}\right)}{\left(I_{0}^{90}+I_{90}^{90}\right)}\right\} .
$$

To estimate distributions $q=\left\{\begin{array}{l}\delta(m, n) \\ \theta(m, n)\end{array}, \quad\right.$ we calculated a set of statistical moments of the $1^{\text {st }}$ to $4^{\text {th }}$ orders $Z_{j=1 ; 2 ; 3 ; 4}$ using the following algorithms [2]

$$
\begin{aligned}
& Z_{1}=\frac{1}{N} \sum_{i=1}^{N}\left|(q)_{i}\right|, Z_{2}=\sqrt{\frac{1}{N} \sum_{i=1}^{N}(q)_{i}^{2}}, \\
& Z_{3}=\frac{1}{\left(Z_{2}\right)^{3}} \frac{1}{N} \sum_{i=1}^{N}(q)_{i}^{3}, Z_{4}=\frac{1}{\left(Z_{2}\right)^{4}} \frac{1}{N} \sum_{i=1}^{N}(q)_{i}^{4} .
\end{aligned}
$$

\section{Experimental results and discussion}

As objects of investigation, we chose two groups of optically-thin (extinction coefficient $\tau \approx 0.090 \ldots 0.095$ ) histological sections of the biopsy taken from benign (26 samples) and malignant (26 samples) tumor of the uterus. The samples were prepared on a freezing microtome by using the standard technique.

\subsection{Spatial frequency Fourier polarimetry of linear birefringence of the fibrillar matrices of the uterine tissue}

For the purpose of choosing optimal conditions of spatial-frequency filtration, the following range $\Delta r=$ $2 \ldots 50$ pix of possible dimensions of the vignetting diaphragm. As a criterion for choosing the size of the aperture was both an extreme change in the set of statistical moments of the $1^{\text {st }}$ to $4^{\text {th }}$ order $Z_{j=1 ; 2 ; 3 ; 4}$ (relation (13)) that characterize the distribution $\delta(v, \mu)$. In our case, we obtained the following optimal size $\Delta r=30$ pix. This geometric size was chosen for comparative investigations of optical anisotropy of the fibrillar networks of the uterine tissue with benign and malignant changes (Fig. 1).

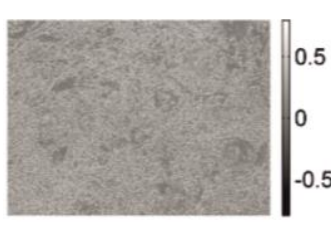

a)

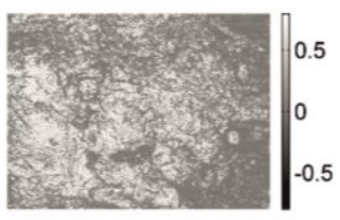

c)
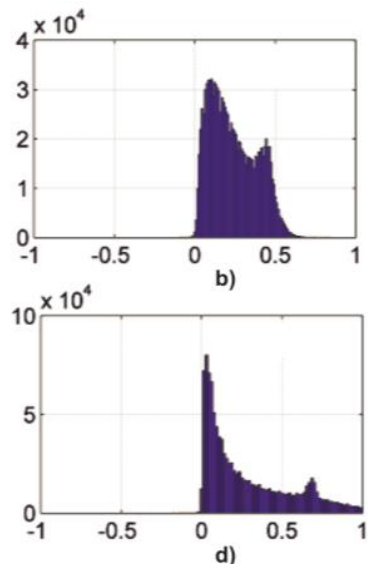

Fig. 1. "Low-frequency" coordinate maps $(a, c)$ and histograms $(b, d)$ of the linear birefringence distribution of a histological section of benign uterine tumor (fibromyoma) biopsy $(a-b)$ and malignant uterine tumor (adenocarcinoma) biopsy $(c-d)$.

Quantitative differences between phase maps of the protein matrices of the uterine tissue of both types illustrate average values and standard deviations of the set of statistic $Z_{i=1 ; 2 ; 3 ; 4}$ parameters, listed in Table 1 . Separate figures are given specificity $S p=a(a+b)^{-1}(a$, $b$ - numbers of correct and incorrect conclusions) of these parameters in differentiation of benign and malignant uterine tumors.

A comparative analysis of the data of Fourier polarimetry of linear birefringence of protein polycrystalline networks of histological section taken from benign and malignant uterine tumors biopsy revealed sensibility to differentiation of these states $\left(Z_{i=1 ; 2 ; 3 ; 4}\right)$ with the specificity level $S p \sim 63 \% \ldots 79 \%$ (printed in grey color in Table 1).

\subsection{Special frequency Fourier polarimetry of circular birefringence of the fibrillar networks of uterine tissue}

Diagnostic possibilities of differentiating the pathologically changed samples of uterine tissue illustrate probable adduced in a series in Fig. 2.

Quantitative differences between phase maps of circular birefringence of the endometrium collagenous networks illustrate average values and standard deviations of the statistic set $Z_{i=1 ; 2 ; 3 ; 4}$ parameters, adduced in Table 2 .

Table 1. Parameters of the statistical, correlative and selfsimilar structure of phase maps for linear birefringence of uterine tissue networks.

\begin{tabular}{|c|c|c|c|}
\hline \multirow{2}{*}{ Parameters } & \multicolumn{2}{|c|}{$\delta(m \times n)$} & \multirow{2}{*}{$S p$} \\
\cline { 2 - 3 } & Fibromyoma & Adenocarcinoma & \\
\hline$Z_{1}$ & $0.37 \pm 0.059$ & $0.47 \pm 0.11$ & $71 \%$ \\
\hline$Z_{2}$ & $0.16 \pm 0.022$ & $0.19 \pm 0.031$ & $63 \%$ \\
\hline$Z_{3}$ & $1.16 \pm 0.37$ & $0.73 \pm 0.14$ & $77 \%$ \\
\hline$Z_{4}$ & $0.79 \pm 0.21$ & $0.57 \pm 0.051$ & $79 \%$ \\
\hline
\end{tabular}


Table 2. Parameters of the statistical, correlative and selfsimilar structure of phase maps for circular birefringence of polycrystalline uterine tissue networks.

\begin{tabular}{|c|c|c|c|}
\hline \multirow{2}{*}{ Parameters } & \multicolumn{2}{|c|}{$\Theta(m \times n)$} & \multirow{2}{*}{$S p$} \\
\cline { 2 - 3 } & Fibromyoma & Adenocarcinoma & \\
\hline$Z_{1}$ & $0.11 \pm 0.021$ & $0.21 \pm 0.037$ & $73 \%$ \\
\hline$Z_{2}$ & $0.14 \pm 0.018$ & $0.23 \pm 0.035$ & $81 \%$ \\
\hline$Z_{3}$ & $0.79 \pm 0.11$ & $1.38 \pm 0.39$ & $86 \%$ \\
\hline$Z_{4}$ & $0.81 \pm 0.13$ & $1.21 \pm 0.21$ & $83 \%$ \\
\hline
\end{tabular}

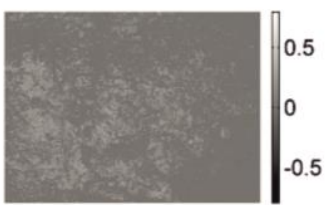

a)

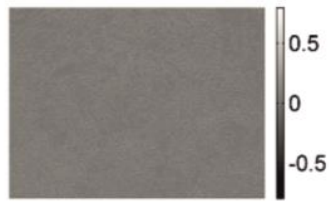

c)
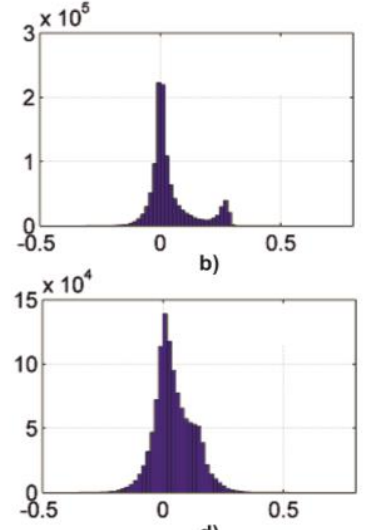

d)
Fig. 2. "High-frequency" coordinate maps $(a, c)$ and histograms $(b, d)$ of the linear birefringence distribution of a histological section of benign uterine tumor (fibromyoma) biopsy $(a-b)$ and malignant uterine tumor (adenocarcinoma) biopsy $(c-d)$.

A comparative analysis revealed the following oncologic condition with a high level of specificity $(73 \% \leq S p \leq 86 \%)$ of uterus parameters (depicted in grey color in Table 2):

- $\quad$ statistical moments of the $1^{\text {st }}$ and $2^{\text {nd }}$ orders $\theta(m \times n)$, differences between the values lies within the range 2 to 3.5 times.

\section{Conclusions}

The model of generalized optic anisotropy in the fibrillar protein matrices has been suggested, and the method of Fourier polarimetry for the parameters of linear and circular birefringence of the tissue biopsy of the uterine wall has been substantiated.

The set of criteria for differentiation of benign (fibromyoma) and malignant (adenocarcinoma) conditions of the uterine has been revealed and substantiated:

- statistical moments of the $1^{\text {st }}-4^{\text {th }}$ orders that characterize distribution of the phase shift between orthogonal components of the laser radiation are defined by linear birefringence of myosin fibers of the endometrium layer;

- statistical moments of the $1^{\text {st }}-4^{\text {th }}$ orders that characterize distribution of plane rotations of laser radiation polarization are defined by circular birefringence of the network of collagenic fibrils of the endometrium layer.

\section{Acknowledgement}

This work was supported by the grants № 0113U003239 and № 0112U002336 from the Ukrainian Foundation for Basic Researches.

\section{References}

1. O.V. Angelsky, A.G. Ushenko, Yu.A. Ushenko, Ye.G. Ushenko, Yu.Ya. Tomka, V.P. Pishak, Polarization-correlation mapping of biological tissue coherent images // J. Biomed. Opt. 10(6), 064025 (2005).

2. Handbook of Coherent-Domain Optical Methods. Biomedical Diagnostics, Environmental and Material Science. Ed. V. Tuchin. Kluwer Academic Publishers, 2004.

3. J.F. de Boer, T.E. Milner, M.J. van Gemert, J.S. Nelson, Two-dimensional birefringence imaging in biological tissue using polarizationsensitive optical coherence tomography // Proc. SPIE, 3196, p. 32-37 (1998).

4. J.F. de Boer, T.E. Milner, M.G. Ducros, S.M. Srinivas and J.S. Nelson, Polarizationsensitive optical coherence tomography, in: Handbook of Optical Coherence Tomography, ed. by B.E. Bouma and G.J. Tearney. Marcel Dekker Inc., New York, 2002, p. 237-274.

5. M.J. Everett, K. Shoenenberger, B.W. Colston, L.B. Da Silva, Birefringence characterization of biological tissue by use of optical coherence tomography // Opt. Lett. 23, p. 228-230 (1998).

6. J.F. de Boer, T.E. Milner, J.S. Nelson, Determination of the depth-resolved Stokes parameters of light backscattered from turbid media by use of polarization-sensitive optical coherence tomography // Opt. Lett. 24, p. 300-302 (1999).

7. A.G. Ushenko, Polarization structure of biospeckles and the depolarization of laser radiation // Optika i Spektroskopiya, 89(4), p. 597600 (2000), in Russian.

8. A.G. Ushenko, Laser probing of biological tissues and the polarization selection of their images// Optika i Spektroskopiya, 91(6), p. 932-936 (2001), in Russian.

9. S. Yermolenko, A. Ushenko, P. Ivashko, F. Goudail, I. Gruia, C. Gavrilă, D. Zimnyakov, A. Mikhailova, Spectropolarimetry of cancer change of biotissues // Proc. SPIE, 7388, 7388 1D, (2009).

10. A. Ushenko, S. Yermolenko, A. Prydij, S. Guminetsky, I. Gruia, O. Toma, K. Vladychenko, Statistical and fractal approaches in laser polarimetry diagnostics of the cancer prostate tissues // Proc. SPIE, 7008, 7008 2C (2008). 
11. S.H. Guminetskiy, A.G. Ushenko, I.P. Polyanskiy, A.V. Motrych, F.V. Grynchuk, The optical method of the investigation of peritonitis progressing process // Proc. SPIE, 7008, 700827 (2008).

12. A.G. Ushenko, Polarization correlometry of angular structure in the microrelief pattern of rough surfaces // Optika i Spektroskopiya, 92, p. 227-229 (2002), in Russian.

13. O.V. Angelsky, A.G. Ushenko, A.D. Arkhelyuk, S.B. Ermolenko, D.N. Burkovets, Structure of matrices for the transformation of laser radiation by biofractals // Quantum Electron. 29, p. 1074-1077 (1999).

14. J.W. Goodman, Statistical properties of laser speckle patters. In: Laser Speckle and Related Phenomena, ed. J.C. Dainty. Berlin, SpringerVerlag, 1975, p. 9-75.

15. S.C. Cowin, How is a tissue built? // J. Biomed. Eng. 122, p. 553-568 (2000).

16. O.V. Angelsky, A.G. Ushenko, Y.G. Ushenko, Complex degree of mutual polarization of biological tissue coherent images for the diagnostics of their physiological state // J. Biomed. Opt. 10(6), 060502 (2005).

17. O.V. Angel'skii, O.G. Ushenko, D.N. Burkovets, O.D. Arkhelyuk, Yu.A. Ushenko, Polarizationcorrelation studies of multifractal structures in bio tissues and diagnostics of their pathologic changes // Laser Phys. 10(5), p. 1136-1142 (2000).

18. A. Gerrard, J.M. Burch, Introduction to Matrix Methods in Optics. New York, A Wiley-Intersci. Publication, 1975.

19. M. Born, E. Wolf, Principles of Optics. Cambridge Univ. Press, Cambridge, 1999.

20. O.V. Angelsky, P.V. Polyanskii, C.V. Felde, The emerging field of correlation optics // Optics and Photonics News, 23(4), p. 25-29 (2012).

21. O.V. Angelsky, A.Ya. Bekshaev, P.P. Maksimyak, A.P. Maksimyak, S.G. Hanson, C.Yu. Zenkova, Self-diffraction of continuous laser radiation in a disperse medium with absorbing particles // Opt. Exp. 21(7), p. 8922-8938 (2013).
22. O.V. Angelsky, G.V. Demianovsky, A.G. Ushenko, D.N. Burkovets, Y.A. Ushenko, Wavelet analysis of two-dimensional birefringence images of architectonics in biotissues for diagnosing pathological changes // J. Biomed. Opt. 9(4), p. 679-690 (2004).

23. O.V. Angel'skii, A.G. Ushenko, S.B. Ermolenko, D.N. Burkovets, Yu.A. Ushenko, O.V. Pishak, Polarization-based visualization of multifractal structures for the diagnostics of pathological changes in biological tissues // Optika $i$ Spektroskopiya, 89(5), p. 799-804 (2000), in Russian.

24. O.V. Angelsky, Yu.A. Ushenko, O.V. Dubolazov, $\mathrm{O} . \mathrm{Yu}$. Telenha, The interconnection between the coordinate distribution of Mueller-matrixes images characteristic values of biological liquid crystals net and the pathological changes of human tissues // Adv. Opt. Technol. 2010, Article ID 130659, 10 pages (2010).

25. Yu.O. Ushenko, Yu.Ya. Tomka, O.V. Dubolazov, V.O. Balanets'ka, A.V. Karachevtsev, A.P. Angelsky, Wavelet-analysis for laser images of blood plasma // AECE - Adv. in Electr. and Comput. Eng. 11(2), (2011)

26. V.T. Bachinsky, Yu.O. Ushenko, Yu.Ya. Tomka, O.V. Dubolazov, V.O. Balanets'ka, A.V. Karachevtsev, Wavelet analysis for polarization maps of networks formed by liquid biological crystals in blood plasma: Statistical and fractal approaches // Semiconductor Physics, Quantum Electronics \& Optoelectronics, 13(2), p. 189-201 (2010).

27. Yu.A. Ushenko, Yu.Ya. Tomka, A.V. Dubolazov, et al., Mueller-matrix diagnostics of optical properties inherent to polycrystalline networks of human blood plasma // Semiconductor Physics, Quantum Electronics \& Optoelectronics, 14(1), p. 98-105 (2011)

28. Yu.O. Ushenko, O.V. Dubolazov, A.O. Karachevtsev, M.P. Gorsky, Yu.F. Marchuk, Wavelet analysis of Fourier polarized images of the human bile // Appl. Opt. 51, p. C133-C139 (2012). 